Original Research Paper

\title{
Description of a Novel Probiotic Concept: Implications for the Modulation of the Immune System
}

\author{
Stefania Pacini and Marco Ruggiero \\ Silver Spring Sagl, Arzo-Mendrisio, Switzerland
}

\section{Article history}

Received: 30-12-2016

Revised: 11-01-2017

Accepted: 19-01-2017

Corresponding Author: Marco Ruggiero

Silver Spring Sagl, Arzo-

Mendrisio, Switzerland

Email:pacini68@yahoo.com

\begin{abstract}
It is estimated that the first processed food products consumed by humans pertained to the category of fermented foods and beverages such as beer, wine, sauerkraut, fermented sausage as well as fermented milk derivatives such as yogurt and kefir. Thus, fermented foods are endowed with undisputed healthy properties due to the presence of probiotic microbes that not only help supporting the healthy human microbiota, but also produce bioactive or bio-available metabolites that contribute to the host's wellbeing. It is widely acknowledged that modulation of the immune system is one of the beneficial effects associated with consuming fermented food products rich in live probiotics and this effect is being exploited in the field of natural immunotherapy. Here we describe the genesis and development of a novel, non-dairy product that may represent a novelty in the field of probiotic drinks since it combines the healthy properties of dairy-based products with the properties of non-dairy probiotic drinks. The major improvements over the existing non-dairy probiotic drinks consist in the number and diversity of probiotic strains endowed with healthpromoting properties and in the presence of a glycosaminoglycan, chondroitin sulfate, that is, at the same time, a newly identified prebiotic and a supplement known to modulate the immune system.
\end{abstract}

Keywords: Probiotic, Immune System, Gut Microbiota, Bifidobacterium, Chondroitin Sulfate

\section{Introduction}

Although the colloquial use of the term "probiotic" by the general public is relatively recent, the concept of "useful" as opposed to "harmful" microbes is more than 100 years old and dates back to Nobel Laureate Élie Metchnikoff who attributed the longevity of Bulgarian peasants to the consumption of fermented milk products such as yogurts and kefirs (Gasbarrini et al., 2016). A search for the term "probiotic" in the database PubMed performed in December 2016, yielded 17,741 papers in many different areas of research. Thus, the list of papers on probiotic encompasses subdivisions as: "Probiotics antibiotic, probiotics prevention, probiotics diarrhea, probiotics immune, probiotics obesity, probiotics irritable bowel, probiotics children, probiotics atopic, probiotics gut, probiotics urinary".

Although a comprehensive review of the effects of probiotics in human health is a monumental task - there exist 4,285 reviews on the topic - it is worth noticing that there is general consensus and solid scientific evidence for an overall beneficial effect of probiotics even in areas that are not directly related with gut function and the immune system. For example, a very recent systematic review on the effect of probiotics on central nervous system functions in animals and humans reported that "probiotics showed efficacy in improving psychiatric disorder-related behaviors including anxiety, depression, Autism Spectrum Disorder (ASD), obsessive-compulsive disorder and memory abilities, including spatial and non-spatial memory" (Wang et al., 2016). In this study, the Authors reviewed 38 randomized controlled trials, 25 of which were performed in animals and 15 in humans. In the reviewed studies, the microbial strains most frequently used were Bifidobacteria and Lactobacilli, administered for 2 weeks in animals and 4 weeks in humans.

Because of different regulations in different parts of the world, the term probiotic has not always the same identical meaning and also the claims that can, or cannot, 
be made for probiotics are different in accordance with local laws and regulations. Such differences render sometimes difficult to compare results and may generate confusion among researchers and consumers as well. For example, the term probiotic is used indifferently for lyophilized microbes that are ingested as powders as well as for fermented products where the live microbes proliferate in their own liquid environment such as yogurts, kefirs or fermented drinks or teas such as kombucha (Greenwalt et al., 2000).

The two main forms of probiotics quoted abovelyophilized microbes and fermented foods or drinksdiffer greatly in their properties, in particular when their effects on the immune system are considered. Thus, Irvine et al. (2010) demonstrated that consumption for about 3 years of a probiotic yogurt supplemented withthat is in addition to the common fermenting strains of yogurts -Lactobacillus rhamnosus, increased the number of CD4 cells in subjects living with HIV/AIDS. These very encouraging results, however, were not replicated when the same identical probiotic strain (Lactobacillus rhamnosus) was administered as an encapsulated probiotic and not as a component of a yogurt. In other words, the probiotic Lactobacillus rhamnosus appeared to stimulate the immune system only when it was a component of a yogurt, thus lending credit to the hypothesis that it was the yogurt with its array of microbes and metabolites produced by the microbes and not the single Lactobacillus rhamnosus strain, responsible for the immune stimulating effect (Hummelen et al., 2011).

Such a striking difference could be explained considering that during the fermentation of milk by the probiotic strains that compose a yogurt, several newly formed peptides are generated and it is likely that the overall immune-reconstituting effect is due to a combination of microbes and the products of their metabolism rather than to the microbes alone (Ebner et al., 2015). Consistent with this interpretation, the Authors concluded that: "The inefficacy of the probiotic strains in preserving the immune function may be the result of using encapsulated probiotics versus the use of probiotic yogurt with the same probiotic strains in previous studies ..." (Hummelen et al., 2011).

Taking into consideration these observation, in 2011 we developed the formula for a probiotic drink that was based on the fermentation of milk and colostrum by a proprietary array of microbes which included fermenting and non-fermenting strains as well as gastro-resistant probiotics. The efficacy of this product in restoring the immune system was first reported in a preliminary study in 2011 (Pacini et al., 2011) and subsequently the product was utilized in the context of the integrated nutritional approach to immunotherapy (Ruggiero et al., 2014a; 2014b; Thyer et al., 2014; Ward et al., 2014; Branca et al., 2015; Schwalb et al., 2016).

In the pursue of exploiting the probiotic features of microbial communities and their metabolites independently of the growth medium, we studied the feasibility of developing a formula similar in principle to that described above, but without the use of milk and colostrum as media for fermentation.

Here, we describe the genesis and development of a non-dairy fermented product that it is based upon our previous experience with the probiotic fermented milk product described above. The starting point for the development of this product that may represent a novel concept in probiotic drinks, is the observation that eukaryotic and prokaryotic probiotic microbes are able to proliferate and ferment in media other than milk (Marsh et al., 2013).

Since the benefits of non-dairy probiotic drinks are well acknowledged and range from immunomodulation to anti-inflammatory and anti-cancer activities (Zamberi et al., 2016), we decided to further enhance these positive aspects by using a known antiinflammatory and immune-modulating glycosaminoglycan, chondroitin sulfate, endowed with prebiotic properties as an addition to the array of probiotic microbes that had proven efficient in the probiotic fermented milk product described above. The rationale for adding chondroitin sulfate lays in its dual role as a prebiotic synergizing with the probiotic strains comprised in the formula and as a supplement endowed with a number of healthy properties (Shang et al., 2016; Ruggiero et al., 2016).

\section{Materials and Methods}

The non-dairy fermented product described in this article is manufactured in Europe under the supervision of the Swiss company Silver Spring Sagl that is the same company that manufactures the probiotic fermented milk product quoted in Schwalb et al. (2016).

The individual components that are necessary to prepare the non-dairy product are manufactured in Europe by companies leaders in their respective fields. These companies are quality certified in conformance with ISO 9001:2008 and, when applicable, in compliance with the rules governing medical products in the European Union laid down in Commission Directives 91/356/EEC, as amended by Directive 2003/94/EC and 91/412/EEC (GMP certification according to the European Pharmacopoeia and the Japanese Pharmacopoeia).

It is well known that fermented drinks and foods may contain alcohol (ethanol) that is an inherent by-product of practically all types of fermentation. In order to precisely assess the amount of alcohol deriving from the 
fermentation process inherent to the present formula, the analyses concerning the amount of alcohol were performed by the Swiss laboratory TIBIO Sagl that represents SCITEC Research SA, Lausanne and is certified by Swiss Medic, State of New York Department of Health, Swiss Testing Labs, Swiss Accreditation STS 0278 and is also quality certified ISO 17025.

The non-dairy fermented product was prepared according to the following procedure.

One liter of non-chlorinated potable water was added with 5 grams of sodium chondroitin sulfate, USP food grade, one sachet of ad hoc formulated fermenting starter enriched in Bifidobacteria, one sachet containing $80 \times 10^{9}$ viable cells of gastro-resistant probiotics (comprising, among others, Bifidobacteria, Lactobacilli, including rhamnosus etc.) and maltodextrin (as one of the prebiotics of the formula), 18 grams of organic sucrose, $17 \mathrm{ml}$ of organic lemon juice, one organic dry prune (approximate weight, 15 grams). This mixture was mixed in a large glass bowl with an ample surface, loosely covered with a paper towel and incubated at room temperature $\left(22^{\circ} \mathrm{C}\right)$ for $24 \mathrm{~h}$.

\section{Results}

Alcohol (ethanol) production during the fermentation of sugar-based beverages may represent a problem for drinks that are not intended as alcoholic beverages and their influence on blood alcohol level has been questioned in a legal proceeding (Rabl et al., 1994). Thus, in 1994, experiments performed at the Swiss Institut für Rechtsmedizin des Kantonsspitals, St. Gallen, demonstrated that a non-dairy probiotic drink formulation, albeit very different from that presented here, led to appreciable ethanol formation that would have qualified the beverage as a bona fide alcoholic drink. Such a formulation was composed of 21 table spoons of water (approximately $310 \mathrm{~mL}$ ), 6 table spoons of kefir granules (approximately 120 grams), 150 grams of sucrose, 2 figs and one lemon. The Authors of this study found that one liter of this non-dairy kefir product contained up to 38 grams per liter of ethanol after 7 to 10 days (Rabl et al., 1994).

In order to avoid these potential problems, the amount of sucrose in our formulation is much lower and the microbial strains were carefully selected in order to avoid the production of ethanol and to guarantee stability. Analyses using gas-chromatography-mass spectrometry demonstrated that the amount of ethanol present one week after the fermentation was below 0.1 grams per liter, thus well below the limit for alcoholic beverages and even below the level of ethanol found in common foods. Thus, researchers at the University of Kaiserslautern in Germany, determined that orange, apple and grape juice contain significant amounts of ethanol (up to 0.77 grams per liter) and certain packed bakery products such as burger rolls or sweet milk rolls contain more than 1.2 grams of ethanol per 100 grams of product (Gorgus et al., 2016).

The measured $\mathrm{pH}$ of the product after $24 \mathrm{~h}$ fermentation, was 3.95 that is a value consistent with an efficient process of fermentation.

The microbiological analyses performed on this novel, non-dairy fermented product showed that the number of Colony Forming Units (CFU) after $24 \mathrm{~h}$ fermentation was $1.2 \times 10^{9}$ per $100 \mathrm{~mL}$ that corresponds to 12 billion CFU per liter. This value of course does not take into account neither the number of non-fermenting Bifidobacteria nor the number of viable cells of gastroresistant probiotics; thus, the total number of probiotics (CFU of fermenting microbes plus Bifidobacteria and gastro-resistant probiotics) is close to $100 \times 10^{9}$ per liter. Please notice that these numbers refer to probiotic microbes that are the only microbes present in the product. In fact, analyses carried out to ascertain the presence of contaminating pathogenic microbes ruled out their presence, thus confirming the safety of the formula and of the fermentation procedure as reported in Table 1.

In detail, analysis for Enterobacteriaceae was performed according to the method designated ISO 21528-2:2004 and showed less than 1 CFU per ml. The methods identified by the suffix "ISO" (International Organization for Standardization) are those designed by the worldwide federation of national standards bodies (ISO member bodies). In particular, ISO 21528-2:2004 is a method for the count of Enterobacteriaceae without pre-enrichment. This method is performed by counting colonies in a solid medium after incubation at $37^{\circ} \mathrm{C}$ and it is used to assess the presence of pathogens in products intended for human consumption as well as in the areas of food production and food handling. In this method, Enterobacteriaceae are defined as microorganisms that form characteristic colonies on violet red bile glucose agar and that ferment glucose and show a negative oxidase reaction when the tests are carried out in accordance with the techniques described in ISO 21528-2:2004.

Table 1. Analysis of pathogen contaminants

\begin{tabular}{ll}
\hline Pathogen & Result \\
\hline Enterobacteriaceae & $<1 \mathrm{CFU} / \mathrm{mL}$ \\
Microbiology & \\
ISO 21528-2:2004 & Zero CFU/100 mL \\
Enterococci & \\
Membrane Filtration & Zero CFU/100 mL \\
ISO 7899-2:2000 & \\
Escherichia Coli & \\
Membrane Filtration & Absence in $25 \mathrm{~mL}$ \\
ISO 9308-1:2000/Cor1:2007 & \\
Listeria monocytogenes & \\
Real-time PCR & \\
AFNOR BRD 07/10-04/05 &
\end{tabular}


Analysis for Enterococci using membrane filtration according to ISO 7899-2:2000, showed the absence of Enterococci; in other words, there was no formation of CFU. ISO 7899 is a method for the detection and count of intestinal Enterococci in water or in other drinkable liquids by membrane filtration. It is worth noticing that this method was originally intended for examination of drinking water, water from swimming pools and other disinfected or clean waters that is for the examination of large volumes of water containing only a few intestinal Enterococci, thus highlighting the sensitivity and specificity of the method. In this method, intestinal Enterococci are defined as bacteria which are able to reduce 2,3,5-triphenyltetrazolium chloride to formazan and to hydrolyze aesculin at $44^{\circ} \mathrm{C}$.

Analysis for Escherichia coli (ISO 93081:2000/Cor1:2007), showed no CFU. ISO 9308$1: 2000 /$ Cor $1: 2007$ is a method for the count of Escherichia coli and coliform bacteria that is based on membrane filtration, followed by culture on a chromogenic coliform agar medium and count of the number of target organisms in the sample. Because of its characteristics, this method is particularly suitable for waters or drinkable products with low bacterial numbers that will cause less than 100 total colonies on chromogenic coliform agar.

Analysis for Listeria monocytogenes using real-time PCR according to AFNOR BRD 07/10-04/05, showed absence of the microbe. This method follows the guidelines of the French Association for Standardization; (Association Française de Normalisation or AFNOR) that is the French national organization for standardization and it is in accordance with the International Organization for Standardization. This technique is based on real-time PCR, where specific probes are used to detect the DNA during the amplification, by hybridizing to the amplicons. This method allows a simple determination of the presence of Listeria monocytogenes in a sample and it is recognized by certification bodies as suitable for the detection of the pathogen in foods or drinks for human consumption.

\section{Discussion}

The non-dairy product described in this study may represent a novelty in the field of probiotic drinks since it combined the healthy properties of dairy-based products with the properties of non-dairy probiotic drinks. The major improvements over the existing nondairy probiotic drinks are the following.

The amount of live microbes; typically, a commercial non-dairy probiotic drink contains about $1 \times 10^{9}$ probiotic microbes per serving, whereas the product described here contains more than $9 \times 10^{9}$ probiotic microbes as calculated by adding the number of fermenting microbes to the number of non-fermenting microbes (e.g., Bifidobacteria) and gastro-resistant probiotics.

The presence of gastro-resistant probiotics, including Bifidobacteria, that are intended to colonize the gut after having passed undamaged the stomach barrier. Please notice that most non-dairy probiotic drinks do not contain Bifidobacteria neither in their fermenting formulas nor as gastro-resistant probiotics. It is known that Bifidobacteria are among the first microbes to colonize the human gastrointestinal tract and there is ample consensus that they exert positive health benefits on their host with particular reference to the immune system (O'Callaghan and van Sinderen, 2016).

The presence of eukaryotic microbes endowed with probiotic features. Among these, we focused our attention on the Kluyveromyces marxianus. Thus, this microbe is known to decrease the production of proinflammatory cytokines in human enterocyte-like cells as well as in human peripheral blood mononuclear cells, thus reducing local and systemic inflammation (Maccaferri et al., 2012). Kluyveromyces marxianus favors growth and colonization of the human colon by Bifidobacteria, thus synergizing with these probiotics that are highly represented in the product described here. In addition, it increases the production of the short-chain fatty acids acetate and propionate (Maccaferri et al., 2012). Kluyveromyces marxianus also modulates human dendritic cell function in vitro and induces a response dominated by Foxp3+ $\mathrm{T}$ regulatory lymphocytes (Smith et al., 2016). Such a response may bear positive consequences in those conditions characterized by excessive inflammation or uncontrolled proliferation. Thus, the protein designated Foxp3 (forkhead box p3) plays a role not only in regulatory $\mathrm{T}$ cell differentiation, but also in cancer, inhibiting tumorigenesis and increasing cancer cell apoptosis (Tan et al., 2014).

The presence of a glycosaminoglycan, chondroitin sulfate, that is endowed with a number of healthy properties and it is now also considered a prebiotic. Thus, although chondroitin sulfate has been used for decades as a dietary supplement and a symptomatic slow acting drug, only recently it has been demonstrated that it is readily degraded and fermented in vitro by human gut microbes, suggesting that it may act as a prebiotic (Shang et al., 2016). In addition, it is well acknowledged that chondroitin sulfate negatively regulates cancer cell signaling (Willis and Klüppel, 2014) and enhances the activity of conventional chemotherapeutics (Ferro et al., 2012), a finding that is consistent with the observation that sulfated motifs of chondroitin sulfate inhibit triple negative breast cancer (Poh et al., 2015). In a peculiar synergism with Kluyveromyces marxianus, chondroitin sulfate inhibits inflammation (Vallières and du Souich, 2010) and is endowed with immune-stimulating properties consisting in the activation of macrophages without 
inducing the production of pro-inflammatory cytokines (Wrenshall et al., 1999). The number of potential preventive and therapeutic effects of chondroitin sulfate is widely acknowledged. Thus, the prestigious Mayo Clinic reports that there exists scientific evidence for the clinical use of chondroitin sulfate in conditions characterized by inflammation such as osteoarthritis, coronary artery disease, psoriasis, muscle soreness or interstitial cystitis. In addition, the same institution presents a long list of the uses for chondroitin sulfate that are based on tradition or scientific theories. Such a list reads:

"aging, allergies, Alzheimer's disease, amyotrophic lateral sclerosis, antioxidant, antiviral, blood clots, bone healing, breast cancer, burns, cervical disc disease, chest pain, chronic venous ulcers, clogged arteries, colorectal cancer, diabetes, gout, gum disease, headaches, heart attack prevention, heart disease prevention, HIV/AIDS, hyperglycemia, high cholesterol, inflammation, inflammatory bowel disease, joint problems, kidney stones, leukemia, lung cancer, malaria, mouth and throat infections, multiple sclerosis, nerve damage, nerve regeneration, neuroblastoma, osteoporosis, pain, Parkinson's disease, premature birth prevention, quality of life (osteoarthritis), rheumatoid arthritis, snoring, soft tissue injury, spinal cord injury, spine problems, surgery, systemic lupus erythematosus, Temporomandibular Joint Disorder (TMJ), transplants, wound healing" (Ruggiero et al., 2016).

In addition to this long list, it should be noticed that supplements containing chondroitin sulfate could be considered in the prevention and treatment of autism spectrum disorders that are conditions associated with increased urinary excretion of glycosaminoglycans (Endreffy et al., 2016). Thus, it has been demonstrated that chondroitin sulfate, as proteoglycan, is a major component of the peri-neuronal nets that control plasticity (Sorg et al., 2016).

\section{Conclusion}

Here we describe a product that may be considered a novel concept in the category of probiotics since it contains a number of microbial strains that are not commonly found in non-dairy probiotic drinks as well as a glycosaminoglycan, chondroitin sulfate, that is at the same time a powerful supplement and a prebiotic synergizing with the probiotic strains contained in this product. We hypothesize that this novel product may find application in all those conditions where natural modulation of the immune system is sought after.

\section{Acknowledgement}

The Authors wish to thank Ms. Fulvia Gianetta of Silver Spring Sagl for her relentless support and insightful advice.

\section{Author's contribution}

The Authors equally contributed to the genesis and development of the project described in this study and to the writing of the manuscript.

\section{Ethics}

This article is original and contains unpublished material. The corresponding author confirms that the other author has read and approved the manuscript.

\section{Conflict of Interest}

Stefania Pacini, MD $\mathrm{PhD}$, is a consultant for the company Silver Spring Sagl that produces and distributes the probiotics described in this study.

Marco Ruggiero, MD, PhD, owns shares in the company Silver Spring Sagl that produces and distributes the probiotics described in this study. Marco Ruggiero is consultant for the company "dr. reinwald healthcare". Marco Ruggiero is member of the Editorial Board of The American Journal of Immunology and is waived from the Article Processing fee for this contribution; he receives no remuneration for his editorial work.

\section{References}

Branca, J.J., S. Pacini and M. Ruggiero, 2015. Effects of pre-surgical Vitamin D supplementation and ketogenic diet in a patient with recurrent breast cancer. Anticancer Res., 35:5525-32. PMID: 26408720

Ebner, J., A. Așçı Arslan, M. Fedorova, R. Hoffmann and A. Küçükçetin et al., 2015. Peptide profiling of bovine kefir reveals 236 unique peptides released from caseins during its production by starter culture or kefir grains. J. Proteom., 117: 41-57.

DOI: 10.1016/j.jprot.2015.01.005

Endreffy, I., G. Bjørklund, F. Dicső, M.A. Urbina and E. Endreffy, 2016. Acid Glycosaminoglycan (aGAG) excretion is increased in children with autism spectrum disorder and it can be controlled by diet. Metab. Brain Dis., 31: 273-8.

DOI: $10.1007 / \mathrm{s} 11011-015-9745-2$

Ferro, M., G. Giuberti, S. Zappavigna, S. Perdonà and G. Facchini et al., 2012. Chondroitin sulphate enhances the antitumor activity of gemcitabine and mitomycin-C in bladder cancer cells with different mechanisms. Oncol. Rep., 27: 409-15. DOI: $10.3892 /$ or.2011.1526

Gasbarrini, G., F. Bonvicini and A. Gramenzi, 2016. Probiotics history. J. Clin. Gastroenterol., 50: 116-S119. 
Gorgus, E., M. Hittinger and D. Schrenk, 2016. Estimates of ethanol exposure in children from food not labeled as alcohol-containing. J. Anal. Toxicol., 40: 537-42. DOI: 10.1093/jat/bkw046

Greenwalt, C.J., K.H. Steinkraus and R.A. Ledford, 2000. Kombucha, the fermented tea: Microbiology, composition and claimed health effects. J. Food Protect., 63: 976-81.

DOI: 10.4315/0362-028X-63.7.976

Hummelen, R., J. Changalucha, N.L. Butamanya, T.E. Koyama and A. Cook et al., 2011. Effect of 25 weeks probiotic supplementation on immune function of HIV patients. Gut Microbes, 2: 80-5. DOI: $10.4161 /$ gmic. 2.2.15787

Irvine, S.L., R. Hummelen, S. Hekmat, C.W. Looman and J.D. Habbema et al., 2010. Probiotic yogurt consumption is associated with an increase of CD4 count among people living with HIV/AIDS. J. Clin. Gastroenterol., 44: e201-5.

DOI: 10.1097/MCG.0b013e3181d8fba8

Maccaferri, S., A. Klinder, P. Brigidi, P. Cavina and A. Costabile, 2012. Potential probiotic Kluyveromyces marxianus B0399 modulates the immune response in Caco-2 cells and peripheral blood mononuclear cells and impacts the human gut microbiota in an in vitro colonic model system. Applied Environ. Microbiol., 78: 956-64. DOI: 10.1128/AEM.06385-11

Marsh, A.J., O. O'Sullivan, C. Hill, R.P. Ross and P.D. Cotter, 2013. Sequence-based analysis of the microbial composition of water kefir from multiple sources. FEMS Microbiol. Lett., 348: 79-85.

DOI: $10.1111 / 1574-6968.12248$

O'Callaghan, A. and D. van Sinderen, 2016. Bifidobacteria and their role as members of the human gut microbiota. Front Microbiol., 7: 925-925. DOI: 10.3389/fmicb.2016.00925

Pacini, S., T. Punzi, G. Morucci and M. Ruggiero, 2011. Macrophages of the Mucosa-Associated Lymphoid Tissue (MALT) as key elements of the immune response to vitamin $\mathrm{D}$ binding protein-macrophage activating factor. Ital. J. Anat. Embryol., 116: 136-136. DOI: 10.13128/IJAE-10160

Poh, Z.W., C.H. Gan, E.J. Lee, S. Guo and G.W. Yip et al., 2015. Divergent synthesis of chondroitin sulfate disaccharides and identification of sulfate motifs that inhibit triple negative breast cancer. Sci. Rep., 5: 14355-14355. DOI: $10.1038 /$ srep 14355

Rabl, W., B. Liniger, K. Sutter and T. Sigrist, 1994. [Ethanol content of Kefir water]. Blutalkohol, 31: 76-9. PMID: 8204224

Ruggiero, M., E. Ward, R. Smith, J.J. Branca and D. Noakes et al., 2014a. Oleic acid, deglycosylated vitamin D-binding protein, nitric oxide: A molecular triad made lethal to cancer. Anticancer Res., 34: 3569-78. PMID: 24982371
Ruggiero, M., J.J.V. Branca, D. Noakes, M. Gulisano and M. Morucci et al., 2014b. Glycosylated oleic acid/vitamin D-binding protein suppresses HER2 oncogene expression in human breast cancer. Anticancer Res., 34: 5845-5847.

Ruggiero, M., H. Reinwald and S. Pacini, 2016. Is chondroitin sulfate responsible for the biological effects attributed to the GC protein-derived Macrophage Activating Factor (GcMAF)? Med. Hypotheses, 94: 126-31. DOI: 10.1016/j.mehy.2016.07.012

Schwalb, M., M. Taubmann, S. Hines, H. Reinwald and M. Ruggiero, 2016. Clinical observation of a novel, complementary, immunotherapeutic approach based on ketogenic Diet, chondroitin sulfate, vitamin D3, oleic acid and a fermented milk and colostrum product. Am. J. Immunol. DOI: 10.3844/ajisp.2016

Shang, Q., J. Shi, G. Song, M. Zhang and C. Cai et al., 2016. Structural modulation of gut microbiota by chondroitin sulfate and its oligosaccharide. Int. J. Biol. Macromol., 89: 489-98. DOI: 10.1016/j.ijbiomac.2016.04.091

Smith, I.M., A. Baker, J.E. Christensen, T. Boekhout and H. Frøkiær et al., 2016. Kluyveromyces marxianus and Saccharomyces boulardii induce distinct levels of dendritic cell cytokine secretion and significantly different $\mathrm{T}$ cell responses $I n$ Vitro. PLoS One, 11: e0167410-e0167410. DOI: $10.1371 /$ journal.pone.0167410

Sorg, B.A., S. Berretta, J.M. Blacktop, J.W. Fawcett and H. Kitagawa et al., 2016. Casting a wide net: Role of perineuronal nets in neural plasticity. J. Neurosci., 36: 11459-11468. DOI: 10.1523/JNEUROSCI.2351-16.2016

Tan, B., M. Anaka, S. Deb, C. Freyer and L.M. Ebert et al., 2014. FOXP3 over-expression inhibits melanoma tumorigenesis via effects on proliferation and apoptosis. Oncotarget, 5: 264-76. PMID: 24406338

Thyer, L., J.J.V. Branca and M. Taubmann, 2014. Clinical experience of immunotherapy based on oleic acid bound to glycosylated vitamin Dbinding protein in localized and metastatic adenocarcinoma of the pancreas. Anticancer Res., 34: 5847-5849.

Vallières, M. and P. du Souich, 2010. Modulation of inflammation by chondroitin sulfate. Osteoarthritis Cartilage, 18: S1-6. DOI: 10.1016/j.joca.2010.02.017

Wang, H., I.S. Lee, C. Braun and P. Enck, 2016. Effect of probiotics on central nervous system functions in animals and humans: A systematic review. J. Neurogastroenterol. Motil., 22: 589-605. DOI: $10.5056 /$ jnm 16018 
Ward, E., R. Smith, J.J.V. Branca, D. Noakes and G. Morucci et al., 2014. Clinical experience of cancer immunotherapy with oleic acid complexed with deglycosylated vitamin D binding protein. Am. J. Immunol., 10: 23-32. DOI: 10.3844/ajisp.2014.23.32

Willis, C.M. and M. Klüppel, 2014. Chondroitin sulfate$\mathrm{E}$ is a negative regulator of a pro-tumorigenic wnt/beta-catenin-collagen 1 axis in breast cancer cells. PLoS One, 9: e103966-e103966.

DOI: 10.1371/journal.pone.0103966
Wrenshall, L.E., R.B. Stevens, F.B. Cerra and J.L. Platt, 1999. Modulation of macrophage and B cell function by glycosaminoglycans. J. Leukoc Biol., 66: 391-400. PMID: 10496308

Zamberi, N.R., N. Abu, N.E. Mohamed, N. Nordin and Y.S. Keong et al., 2016. The antimetastatic and antiangiogenesis effects of kefir water on murine breast cancer cells. Integr. Cancer Ther., 15: NP53-NP66. DOI: $10.1177 / 1534735416642862$ 